NASA LAW, February 14-16, 2006, UNLV, Las Vegas

\title{
Measurements of Polyatomic Molecule Formation on an Icy Grain Analog Using Fast Atoms
}

\author{
A. Chutjian ${ }^{1}$ S. Madzunkov ${ }^{1}$ B. J. Shortt ${ }^{2}$ J. A. MacAskill ${ }^{1}$ M. R. Darrach ${ }^{1}$ \\ ${ }^{1}$ Jet Propulsion Laboratory/Caltech, Pasadena, CA 91109 \\ ${ }^{2}$ Dept. Applied Physics, Cork Institute of Technology, Cork, Ireland
}

\begin{abstract}
Carbon dioxide has been produced from the impact of a monoenergetic $\mathrm{O}\left({ }^{3} \mathrm{P}\right)$ beam upon a surface cooled to $4.8 \mathrm{~K}$ and covered with a $\mathrm{CO}$ ice. Using temperature-programmed desorption and mass spectrometer detection, we have detected increasing amounts of $\mathrm{CO}_{2}$ formation with $\mathrm{O}\left({ }^{3} \mathrm{P}\right)$ energies of 2 , 5,10 , and $14 \mathrm{eV}$. This is the first measurement of polyatomic molecule formation on a surface with superthermal atoms. The goal of this work is to detect other polyatomic species, such as $\mathrm{CH}_{3} \mathrm{OH}$, which can be formed under conditions that simulate the grain temperature, surface coverage, and superthermal atoms present in shock-heated circumstellar and interstellar regions.
\end{abstract}

\section{Introduction}

Dust, ices, and surfaces of planets, comets, and asteroids play an indispensable role in the chemical evolution of the protostellar regions and the interstellar medium (ISM), from the catalytic production of molecular hydrogen to the formation of organics $\left(\mathrm{CH}_{3} \mathrm{OH}, \mathrm{H}_{2} \mathrm{CO}\right.$, $\mathrm{CH}_{4}, \mathrm{NH}_{3}, \mathrm{CN}$-bonded species, etc.) critical to the origin of life. Laboratory studies of ices and dust grain analogs are crucial to defining and constraining the environmental conditions in dense and dusty clouds. Grain-particle interactions include effects of ion and neutral atomic fluxes, UV radiation intensity, cosmic-ray intensity, and surface temperature. A wide range of laboratory studies has been carried out on effects of ion irradiation; UV photolysis of molecules (including amino acids) on icy grain analogs; thermal-energy atomic irradiation by oxygen atoms, and on thermal desorption characteristics of molecules from analogs of icy dust grains.

Fast neutral species such as $\mathrm{H}, \mathrm{He}, \mathrm{O}, \mathrm{OH}, \mathrm{CO}$, and $\mathrm{H}_{2} \mathrm{O}$ are present in the circumstellar regions and the ISM by virtue of energetic processes such as stellar outflows, shocks, dissociation and ionization of gas-phase particles by stellar UV photons, and the charge 
exchange of outgoing stellar wind ions with circumstellar neutral clouds. Neutral-neutral reactions almost certainly play a major role within cold dark clouds where UV penetration is blocked by dust, and in hot molecular cores where shock heating of neutral atomic species can occur (Ehrenfreund and Charnley 2000). The clearest example of the importance of neutral-neutral reactions is the astrophysical observation that abundant $\mathrm{CO}_{2}$ is detected in a quiescent dark cloud (Elias 16), where no sources of UV radiation are present, and hence where photochemistry cannot have a role (Whittet et al. 1998).

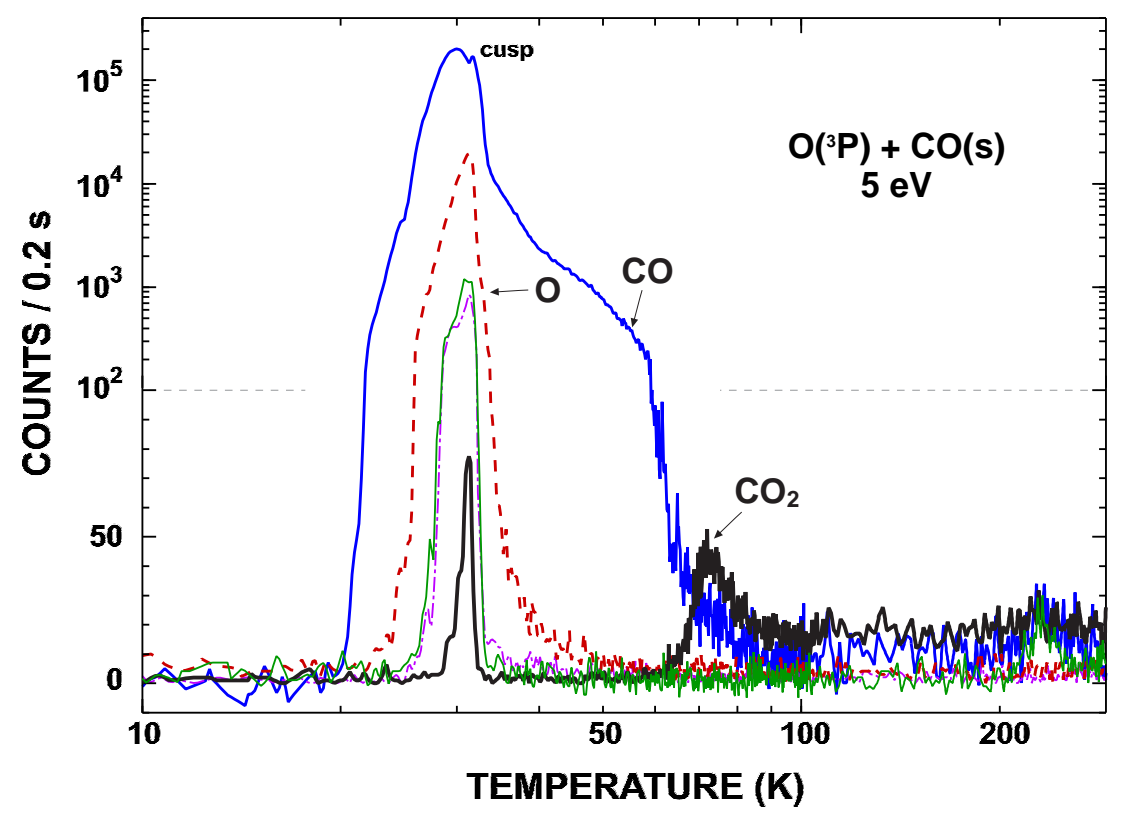

Fig. 1. - TPD spectrum following the exposure to a $5 \mathrm{eV} \mathrm{O}\left({ }^{3} \mathrm{P}\right)$ beam of $\mathrm{CO}$ frozen on a gold substrate. The TPD consists of a low-temperature physisorbed peak $(30 \mathrm{~K})$, and a high temperature $(70 \mathrm{~K})$ chemisorbed peak consisting of only $\mathrm{CO}_{2}$. The mass channels are ${ }^{16} \mathrm{O}$ (broken red line), ${ }^{18} \mathrm{H}_{2} \mathrm{O}$ (solid green line), ${ }^{28} \mathrm{CO}$ (solid blue line), ${ }^{32} \mathrm{O}_{2}$ (broken purple line), and ${ }^{44} \mathrm{CO}_{2}$ (thick solid black line). The peak/valley ratio of the cusp is 0.7 .

\section{Experimental Methods}

The fast-atom apparatus is described in detail in (Madzunkov et al. 2006) [see also (Orient et al. 1992; Orient et al. 1993)]. The surface target consists of CO frozen on a gold surface mounted to a linear translation stage. In a typical temperature-programmed desorption (TPD) run a cleaned gold surface is translated into deposition and bombardment position. There, the surface is cooled to $4.8 \mathrm{~K}$ by a flow of liquid helium, whereupon $\mathrm{CO}$ is introduced into the chamber at a pressure of $10^{-7} \mathrm{~Pa}$ (as measured near the target). The CO molecules are deposited onto the cold surface while the surface is simultaneously bombarded by the $\mathrm{O}\left({ }^{3} \mathrm{P}\right)$ and ground state beam for one hour. The oxygen beams used in separate 
exposures were at energies of $2,5,10$, and $14 \mathrm{eV}$, at neutral currents of $10^{7}$ atoms/sec. The base pressure in the target region is $5 \times 10^{-10} \mathrm{~Pa}$.

After exposure the $\mathrm{O}\left({ }^{3} \mathrm{P}\right)$ beam is turned off. The surface is retracted to position where it is then warmed to $350 \mathrm{~K}$ at a rate of $1.0 \mathrm{~K} / \mathrm{min}$ to $100 \mathrm{~K}$, and at a rate of $10 \mathrm{~K} / \mathrm{min}$ from $100 \mathrm{~K}$ to $350 \mathrm{~K}$. The desorbing species are detected as a function of surface temperature with a quadrupole mass spectrometer (MS). An example of the TPD data is shown in Fig. 2 for $5 \mathrm{eV}$ O-atom energy. Comparable results were obtained at 2, 10, and $14 \mathrm{eV}$. A personal computer is used to monitor and record the electron beam current, target surface temperature, and the mass spectrometer signal for multiple species, while controlling the electron beam energy, electrode potentials, and the surface-target heater. The temperature ramp is sufficiently slow that multiple scans of the MS are performed at each temperature step. The MS is used in a multiple ion monitoring mode to detect the production of up to eight separate masses during the TPD ramp.

\section{Experimental Results}

Five desorbed species are evident in Fig. 1. The strong, cusped peak at $30 \mathrm{~K}$ is interpreted as being due to physisorbed and chemisorbed $\mathrm{CO}$ ice layers, in which the bonding is due to relatively weak CO-CO intermolecular (physisorbed) and strong CO-surface (chemisorbed) potentials. Warming the surface up to the $30 \mathrm{~K}$ melting point of this ice mlange throws all the reacted and adsorbed species into the vapor phase. The ejected species are ${ }^{18} \mathrm{H}_{2} \mathrm{O},{ }^{28} \mathrm{CO},{ }^{32} \mathrm{O}_{2}$, and ${ }^{44} \mathrm{CO}_{2}$. The ${ }^{12} \mathrm{C}$ and ${ }^{16} \mathrm{O}$ arise from fractionation in the MS ionizer of $\mathrm{H}_{2} \mathrm{O}, \mathrm{CO}, \mathrm{O}_{2}$, and $\mathrm{CO}_{2}$. The contribution of ${ }^{28} \mathrm{~N}_{2}$ to the signal at mass 28 was negligible, as evidenced by the fact that no fractionated ${ }^{14} \mathrm{~N}$ could be detected in the baseline vacuum prior to and following each TPD measurement. In addition, the detected $\mathrm{C}^{+} / \mathrm{CO}^{+}$intensity ratio was consistent with that given by the fraction of $\mathrm{CO}$ alone. The source of the $\mathrm{H}_{2} \mathrm{O}$ is trace background water in the vacuum chamber. A search was made in a separate measurement for other mass species in the range 5-50 amu. None was found to within the system detection limit.

At $70 \mathrm{~K}$ one detects only desorption of $\mathrm{CO}_{2}$ chemisorbed to the gold substrate, and formed in the reaction $\mathrm{O}+\mathrm{CO}(\mathrm{s}) \rightarrow \mathrm{CO}_{2}(\mathrm{~s})$. Finally, one detects at $230 \mathrm{~K}$ desorption of the residual $\mathrm{H}_{2} \mathrm{O}$ background frozen on the target (Fig. 1). The $\mathrm{CO}$ desorption temperature range found here agrees with another study in which $\mathrm{CO}$ desorption was found to start at $24 \mathrm{~K}$, and was gone by $33 \mathrm{~K}$ [see also (Sandford and Allamandola 1998)]. The behavior of the CO desorption was used to provide an estimate of approximately a 2-3 monolayer CO coverage, in agreement with an estimate based on the CO partial pressure, exposure time, and a unity sticking coefficient.

A TPD normalization procedure was adopted in order to exhibit the clear enhancement of integrated TPD signals, measured for the different ion masses $\left({ }^{12} \mathrm{C},{ }^{16} \mathrm{O},{ }^{18} \mathrm{H}_{2} \mathrm{O},{ }^{28} \mathrm{CO}\right.$, 


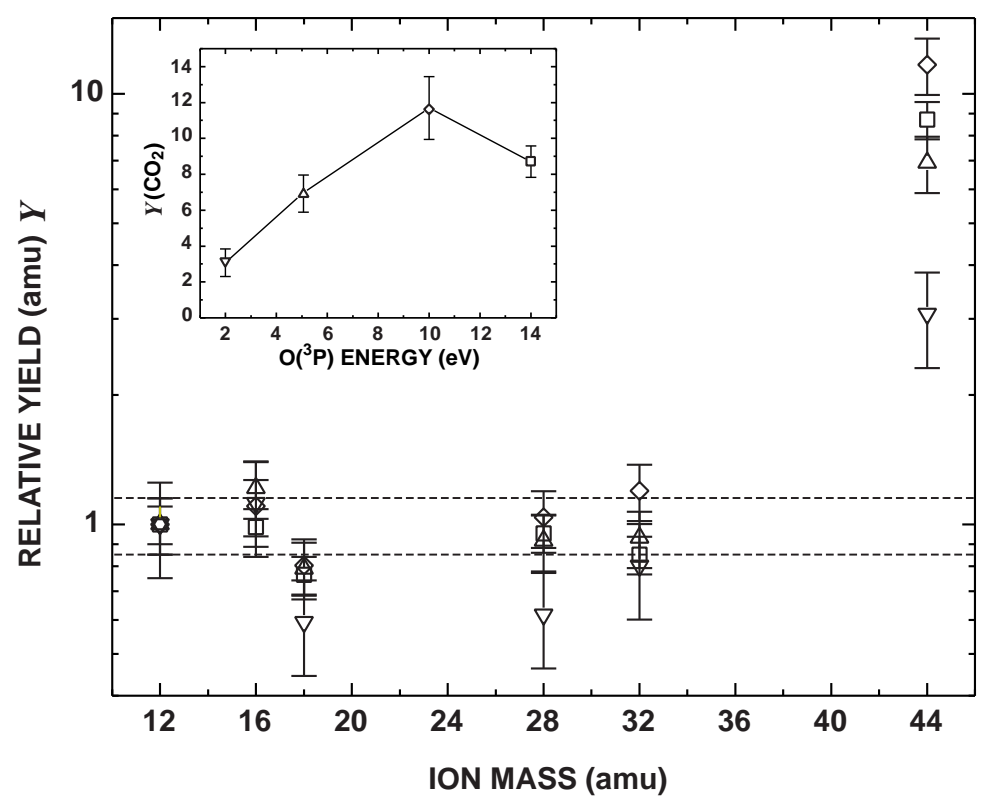

Fig. 2.- Relative production yields $\mathcal{Y}$ of the species ${ }^{12} \mathrm{C},{ }^{16} \mathrm{O},{ }^{18} \mathrm{H}_{2} \mathrm{O},{ }^{28} \mathrm{CO},{ }^{32} \mathrm{O}_{2}$, and ${ }^{44} \mathrm{CO}_{2}$ at $2 \mathrm{eV}(\nabla), 5 \mathrm{eV}(\triangle), 10 \mathrm{eV}(\diamond)$, and $14 \mathrm{eV}(\square)$. Shown in the insert is the excitation function for production of $\mathrm{CO}_{2}$ as a function of the $O\left({ }^{3} P\right)$ energy (Madzunkov et al. 2006).

${ }^{32} \mathrm{O}_{2}$ and ${ }^{44} \mathrm{CO}_{2}$ ), due to O-atom exposure (Madzunkov et al. 2006). From this one can then obtain an expression for the relative yield $\mathcal{Y}$ of species production. Plotted in Fig. 2 are measured values of $\mathcal{Y}$ for each ion mass (with the value of $\mathcal{Y}$ for ${ }^{12} \mathrm{C}$ taken as unity). The horizontal band is the average value $\mathcal{Y}=0.97 \pm 0.1(1 \sigma)$ as measured for the first five masses. Also shown is the error in $\mathcal{Y}$ arising from statistical error, error in measurement of the $\mathrm{CO}$ pressure, and error in knowledge of the laser power within the cavity. One sees clear enhancement in $\mathrm{CO}_{2}$ production (mass 44) at all atomic oxygen energies.

This work was carried out at JPL/Caltech, and was supported by NASA.

\section{REFERENCES}

Ehrenfreund, P. and Charnley, S. B. 2000, Ann. Rev. Astron. Astrophys. 38, 427.

Madzunkov, S., Shortt, B. J., MacAskill, J. A., Darrach, M. R. and Chutjian, A. 2006, Phys. Rev. A 73, 020901.

Orient, O. J., Martus, K. E., Chutjian, A. and Murad, E. 1992, Phys. Rev. A 45, 2998.

Orient, O. J., Chutjian, A., Martus, K. E. and Murad, E. 1993, Phys. Rev. A 48, 427.

Orient O. J. and Chutjian, A. 1999, Phys. Rev. A 59, 4374.

Sandford, A. S. and Allamandola, L. J. 1988, Icarus 765, 201.

Whittet, D. C. B. et al. 1998, Ap. J. 498, L159. 